\title{
Extermal properties of Zagreb coindices and degree distance of graphs
}

\author{
S. Hossein-Zadeh, A. Hamzeh, and A. R. Ashrafi
}




\title{
EXTREMAL PROPERTIES OF ZAGREB COINDICES AND DEGREE DISTANCE OF GRAPHS
}

\author{
S. HOSSEIN-ZADEH, A. HAMZEH AND A. R. ASHRAFI
}

Received 7 December, 2009

\begin{abstract}
The degree distance, Zagreb coindices and reverse degree distance of a connected graph have been studied in mathematical chemistry. In this paper some new extremal values of these topological invariants over some special classes of graphs are determined.
\end{abstract}

2000 Mathematics Subject Classification: 05C35, 05C12, 05A20, 05 C05

Keywords: Zagreb coindex, Zagreb index, degree distance, reverse degree distance

\section{INTRODUCTION}

All graphs in this paper are finite and simple. A graph invariant is a function on a graph that does not depend on the labeling of its vertices. Such quantities are also called topological indices. Two of these graph invariants are known under various names, the most commonly used ones are the first and second Zagreb indices. Due to their chemical relevance they have been subject of numerous papers in the chemical literature $[9,12,13,16,20]$, while the first Zagreb index attracted a significant attention of mathematicians.

The problem of determining extremal values and the corresponding extremal graphs of some graph invariants is the topic of several papers $[2,4,10,13-15,17,20]$. The aim of this paper is to investigate similar problems for a recently introduced generalization of Zagreb indices.

Let $G$ be a connected graph with vertex and edge sets $V(G)$ and $E(G)$, respectively. For every vertex $u \in V(G)$, the edge connecting $u$ and $v$ is denoted by $u v$ and $\operatorname{deg}_{G}(u)$ denotes the degree of $u$ in G. The distance $d_{G}(u, v)$ is defined as the length of a shortest path connecting $u$ and $v$ and the diameter of $G$, $\operatorname{diam}_{G}(G)$, is the maximum possible distance between any two vertices in the graph. We will omit the subscript $G$ when the graph is clear from the context.

This research was in part supported by a grant from IPM (No 88200124). 
The first and second Zagreb indices were originally defined as

$$
M_{1}(G)=\sum_{u \in V(G)} \operatorname{deg}(u)^{2}
$$

and

$$
M_{2}(G)=\sum_{u v \in E(G)} \operatorname{deg}(u) \operatorname{deg}(v),
$$

respectively. The first Zagreb index can be also expressed as a sum over edges of $G, M_{1}(G)=\sum_{u v \in E(G)}[\operatorname{deg}(u)+\operatorname{deg}(v)]$. We refer the reader to [16] for the proof of this fact. The readers interested in more information on Zagreb indices can be referred to $[4,9,10,12,14,16,17]$ and to the references therein.

The Zagreb indices can be viewed as the contributions of pairs of adjacent vertices to certain degree-weighted generalizations of Wiener polynomials [7]. It turned out that computing such polynomials for certain composite graphs depends on such contributions from pairs of non-adjacent vertices.

The first and second Zagreb coindices were first introduced by Došlić. They are defined as follows:

$$
\begin{aligned}
& \bar{M}_{1}(G)=\sum_{u v \notin E(G)}[\operatorname{deg}(u)+\operatorname{deg}(v)], \\
& \bar{M}_{2}(G)=\sum_{u v \notin E(G)}[\operatorname{deg}(u) \operatorname{deg}(v)] .
\end{aligned}
$$

Dobrynin and Kochetova [6] and Gutman [8] introduced a new graph invariant, "degree distance", defined as follows: the degree distance of a vertex $x$, denoted by $D^{\prime}(x)$, is defined as $D^{\prime}(x)=D(x) \operatorname{deg}(x)$, where $D(x)=\sum_{y \in V(G)} d(x, y)$ and the degree distance of $\mathrm{G}$, denoted by $D^{\prime}(G)$, is

$$
\begin{aligned}
D^{\prime}(G) & =\sum_{x \in V(G)} D^{\prime}(x)=\sum_{x \in V(G)} D(x) \operatorname{deg}(x) \\
& =\frac{1}{2} \sum_{x, y \in V(G)} d(x, y)[\operatorname{deg}(x)+\operatorname{deg}(y)] .
\end{aligned}
$$

If $G$ is $n$-vertex graph then the reverse Wiener matrix is an $n \times n$ matrix $R W(G)=$ $\left[R W_{i j}\right]$ such that $R W_{i j}=\operatorname{diam}(G)-d\left(v_{i}, v_{j}\right)$, if $i \neq j$ and 0 otherwise. The reverse degree distance of $G$ is defined as ${ }^{r} D^{\prime}(G)=\sum_{i=1}^{n} \operatorname{deg}\left(v_{i}\right) \sum_{j=1}^{n} R W_{i j}$ [21].

The girth of $G$ is the length of a shortest cycle contained in $G$. A Moore graph is a graph of diameter $k$ with girth $2 k+1$. Those graphs have the minimum number of vertices possible for a regular graph with given diameter and maximum degree. For a real number $k$, let $\sum_{k}(G)$ denote the sum of the $k$-th powers of the degrees of $G$. We denote by $\sigma_{2}(n, m)$ the maximum value of $\sum_{2}(G)$ when $G$ is a graph (not necessarily connected) with $n$ vertices and $m$ edges. Also, let $\sum_{2}(n, m)=\max _{G \in \wp_{n, m}} \sum_{2}(G)$ where $\wp_{n, m}$ denotes the family of connected graphs on $n$ vertices and $m$ edges. 
For the sake of completeness we state here some results from the references $[1,3$, 10,11,14,17,21] which will be useful throughout the paper.

Lemma A (Ashrafi, Došlić and Hamzeh [1]). Suppose $G$ is a connected graph with exactly $n$ vertices and $m$ edges. Then we have:

(a) $\bar{M}_{1}(G)=2 m(n-1)-M_{1}(G)$,

(b) $\bar{M}_{2}(G)=2 m^{2}-M_{2}(G)-\frac{1}{2} M_{1}(G)$.

The subdivision graph $S(G)$ of a graph $G$ is obtained by inserting a new vertex of degree two on each edge of $G$. If $G$ has $n$ vertices and $m$ edges, then $S(G)$ has $n+m$ vertices and $2 m$ edges.

Lemma B (Ilić and Stevanović [10]). Suppose $G$ is a graph with exactly $n$ vertices and $m$ edges. Then

(a) It holds that $M_{1}(G) \geq \frac{4 m^{2}}{n}$. The equality is attained if and only if $G$ is regular.

(b) It holds that $M_{2}(G) \geq \frac{4 m^{3}}{n^{2}}$. The equality is attained if and only if $G$ is regular.

(c) Let $\Delta$ be the maximum vertex degree in $G$. Then $\frac{M_{1}(G)}{n} \leq \frac{\Delta M_{1}(G)}{2 m}$ and $\frac{M_{2}(G)}{m} \leq \frac{\Delta M_{1}(G)}{2 m}$. Equality is attained simultaneously in both inequalities if and only if $G$ is regular.

(d) $M_{1}(S(G))=M_{1}(G)+4 m$ and $M_{2}(S(G))=2 M_{1}(G)$.

Lemma C (Liu and Liu [14]). Let $G$ be a connected graph with $n$ vertices and $m$ edges. Then

(a) If $\Delta \geq \frac{2 m-\delta(n-1)}{2}$, then $M_{1}(G) \leq m(\Delta+\delta+1)$.

(b) $M_{1}(G) \leq \frac{(\Delta+\delta)^{2}}{n \Delta \delta} m^{2}$ with equality if and only if $G$ is regular.

Lemma D (Sun and Wei [17]). If $G$ is a connected bicyclic graph with exactly $n$ vertices, $m$ edges and without pendant vertices, then $\frac{M_{1}(G)}{n} \leq \frac{M_{2}(G)}{m}$, with equality if and only if $G=K_{2,3}$.

Lemma $\mathbf{E}$ (Zhou and Trinajstić [21]). Let $G$ be a connected graph with $n$ vertices, $m$ edges and diameter $l$. Then

(a) ${ }^{r} D^{\prime}(G)=2 m(n-1) l-D^{\prime}(G)$.

(b) If $n \geq 2$ then $(l-1) M_{1}(G) \leq{ }^{r} D^{\prime}(G) \leq 2 m(l-2)(n-1)+M_{1}(G)$ with either equality if and only if $l \leq 2$.

(c) Let $G$ be a connected triangle-free and quadrangle-free graph with $n \geq 3$ vertices, minimal degree $\delta$ and maximal degree $\Delta$. Then $[l-1+\delta(l-2)] M_{1}(G)$ $-2 m(l-2) \delta \leq{ }^{r} D^{\prime}(G) \leq(\Delta+2) M_{1}(G)+2 m[(l-3)(n-1)-\Delta]$ with right equality if and only if $G$ is a Moore graph of diameter 2 , or a regular graph of diameter 3 (and girth 5, 6 or 7) and with left equality if and only if $G=S_{n}$, or $G$ is a Moore graph of diameter 2, or a regular graph of diameter 3 (and girth 5, 6 or 7 ). 
(d) $M_{1}(G) \leq n(n-1)$ with equality if and only if $G$ is the star or a Moore graph of diameter 2 .

(e) Let $G$ be a connected graph with minimal degree $\delta$ and maximal degree $\Delta$. Then $2 l(n-1) m-2 \Delta W(G) \leq{ }^{r} D^{\prime}(G) \leq 2 l(n-1) m-2 \delta W(G)$ with either equality if and only if $G$ is a regular graph.

(f) Let $G$ be a tree with $n$ vertices and diameter $\operatorname{diam}(G)$. Then ${ }^{r} D^{\prime}(G)=$ $2(n-1)^{2} \operatorname{diam}(G)-4 W(G)+n(n-1)$.

The join $G+H$ of graphs $G$ and $H$ with disjoint vertex sets $V(G)$ and $V(H)$ and edge sets $E(G)$ and $E(H)$ is the graph union $G \cup H$ together with all the edges joining $V(G)$ and $V(H)$.

Lemma $\mathbf{F}$ (Bucicovschi and Cioabă [3]). If $1 \leq n-1 \leq m$ and $G$ is a connected graph with $n$ vertices and $m$ edges, then $D^{\prime}(G) \geq 4(n-1) m-\sum_{2}(n, m)=4(n-$ 2) $m-n^{2}+5 n-4-\sigma_{2}(n-1, m-n+1)$. Equality happens if and only if $G$ is $a$ join of $K_{1}$ and a graph $G^{\prime}$ on $n-1$ vertices and $m-n+1$ edges with $\sum_{2}\left(G^{\prime}\right)=$ $\sigma_{2}(n-1, m-n+1)$.

Let $\wp_{n}^{1}$ and $\wp_{n}^{2}$ denote the classes of connected unicyclic and bicyclic graphs, respectively. Note that any graph in $\wp_{n}^{1}$ contains a unique cycle and it has $n$ edges and every graph in $\wp_{n}^{2}$ contains two linearly independent cycles, cycles without common edges, having $n+1$ edges.

Lemma G (Tomescu [11]). Suppose $G$ is a connected graph with $n$ vertices, $m$ edges. Then we have:

(a) For every $n \geq 3$ we have $\min _{G \in \wp_{n}^{1}} D^{\prime}(G)=3 n^{2}-3 n-6$ and the unique extremal graph is $K_{1, n-1}+e$.

(b) For every $n \geq 4$ we have $\min _{G \in \wp_{n}^{2}} D^{\prime}(G)=3 n^{2}+n-18$. The extremal graph is unique and may be obtained from $K_{1, n-1}$ by adding two edges having a common extremity.

Throughout this paper our notation is standard. For terms and concepts not defined here we refer the reader to any of several standard monographs such as, e.g., [5] or [18].

\section{Results}

In this section some new extremal values of Zagreb coindices, degree distance and reverse degree distance over some special classes of graphs are determined. We begin by Zagreb coindices of graphs.

\subsection{Zagreb coindices}

The aim of this subsection is to obtain new lower and upper bounds for the first and second Zagreb coindices of graphs.

Proposition 1. Let $G$ be a simple graph with $n$ vertices and $m$ edges. Then $\bar{M}_{1}(G) \leq \frac{-4 m^{2}}{n}+2 m(n-1)$ with equality if and only if $G$ is regular. 
Proof. Apply Lemma A(a) and Lemma B(a).

Proposition 2. Let $G$ be a simple graph with $n$ vertices, $m$ edges. Then $2 m^{2}-$ $\frac{M_{1}(G)}{2}(\Delta+1) \leq \bar{M}_{2}(G) \leq 2 m^{2}\left(1-\frac{2 m}{n^{2}}-\frac{1}{n}\right)$. The right hand (left hand) side of this inequality is satisfied if and only if $G$ is regular.

Proof. The right hand side is a consequence of Lemma A(b) and Lemma B(b). To prove the left hand side of inequality, we notice that by Lemma A and Lemma B(c), we have:

$$
\begin{aligned}
\frac{\bar{M}_{2}(G)}{m} & =2 m-\frac{M_{2}(G)}{m}-\frac{1}{2 m} M_{1}(G) \\
& \geq 2 m-\frac{\Delta M_{1}(G)}{2 m}-\frac{1}{2 m} M_{1}(G) \\
& =2 m^{2}-\frac{M_{1}(G)}{2}(\Delta+1),
\end{aligned}
$$

which completes our argument. By Lemma A and Lemma B(c) the equality holds if and only if $G$ is regular.

Proposition 3. Let $G$ be a simple graph with $n$ vertices, $m$ edges.

(a) $\bar{M}_{1}(S(G)) \leq 4 m(n-2)+4 m^{2}\left(1-\frac{1}{n}\right)$, the equality holds if and only if $G$ is regular.

(b) $\bar{M}_{2}(S(G)) \leq 8 m^{2}-2 m-\frac{10 m^{2}}{n}$, the equality holds if and only if $G$ is regular.

(c) $\bar{M}_{2}(S(G))=-2 m^{2}+18 m-10 m n+\frac{5}{2} \bar{M}_{1}(S(G))$.

Proof. The parts (a) and (b) are obtained from Lemma A and Lemma B(a) and the following inequalities:

$$
\begin{aligned}
\bar{M}_{1}(S(G)) & =4 m(n+m-1)-M_{1}(S(G)) \\
& =4 m(n+m-1)-M_{1}(G)-4 m \\
& =4 m(n+m-2)-M_{1}(G) \\
& \leq 4 m n+4 m^{2}-8 m-\frac{4 m^{2}}{n} \\
& =4 m(n-2)+4 m^{2}\left(1-\frac{1}{n}\right),
\end{aligned}
$$

and,

$$
\begin{aligned}
\bar{M}_{2}(S(G)) & =8 m^{2}-M_{2}(S(G))-\frac{1}{2} M_{1}(S(G)) \\
& =8 m^{2}-2 m-\frac{5}{2} M_{1}(G) \\
& \leq 8 m^{2}-2 m-\frac{10 m^{2}}{n}
\end{aligned}
$$


The equalities in parts (a) and (b) are also obtained from Lemma A and Lemma B(a). For part (c), we apply Lemma A, Lemma B(d) and the following equality:

$$
\begin{aligned}
\bar{M}_{2}(S(G)) & =8 m^{2}-2 m+\frac{5}{2}\left(-4 m n-4 m^{2}+8 m+\bar{M}_{1}(S(G))\right) \\
& =-2 m^{2}+18 m-10 m n+\frac{5}{2} \bar{M}_{1}(S(G)) .
\end{aligned}
$$

This completes our proof.

Proposition 4. Suppose $G$ is a graph with $n$ vertices and $m$ edges. Then $\bar{M}_{1}(G)$ $\geq 2 m(n-1)-m(\Delta+\delta+1)$.

Proof. Apply Lemma A(a) and Lemma C(a).

Proposition 5. Suppose $G$ is a connected graph with $n$ vertices, $m$ edges. $\bar{M}_{1}(G) \geq 2 m(n-1)-\frac{(\Delta+\delta)^{2}}{n \Delta \delta} m^{2}$. The equality holds if and only if $G$ is regular.

Proof. Apply Lemma A(a) and Lemma C(b).

Proposition 6. Let $G$ be a connected bicyclic graph with $n$ vertices and $m$ edges without pendant vertices, then $\frac{\bar{M}_{1}(G)}{n} \geq \frac{2 m(n-1)}{n}+\frac{\bar{M}_{2}(G)}{m}-2 m+\frac{M_{1}(G)}{2 m}$ with equality if and only if $G$ is isomorphic to $K_{2,3}$.

Proof. By Lemmas A and D $\frac{\bar{M}_{1}(G)}{n}=\frac{2 m(n-1)}{n}-\frac{M_{1}(G)}{n} \geq \frac{2 m(n-1)}{n}-\frac{M_{2}(G)}{m}$ and $\frac{\bar{M}_{2}(G)}{m}=2 m-\frac{M_{2}(G)}{m}-\frac{M_{1}(G)}{2 m}$, as desired.

Proposition 7. Let $G$ be a graph of order $n$ containing $m$ edges, then $2\left(m^{2}+\right.$ $\left.\bar{m}^{2}\right)-\left(\begin{array}{c}n \\ 2\end{array}\right)(n-1)^{2}-\frac{n(n-1)^{2}}{2} \leq \bar{M}_{2}(G)+\bar{M}_{2}(\bar{G}) \leq 2\left(m^{2}+\bar{m}^{2}\right)-\left(\begin{array}{c}n \\ 2\end{array}\right)\left(\frac{n-1}{2}\right)^{2}-\frac{n(n-1)^{2}}{2}$. The equality in right hand side is satisfied if and only $n \cong 1(\bmod 4)$ and $G$ is $\frac{n-1}{2}$ regular. The equality in left hand side is satisfied if and only $G$ is isomorphic to complete graph $K_{n}$.

Proof. Apply [19, Theorems 2.2 and 3.1].

\subsection{Degree Distance of Graphs}

The degree distance of a graph is a degree analogue of the Wiener index of the graph. So, it is useful to find the lower and upper bounds of this topological index on some classes of graphs as trees, triangle- and quadrangle-free graphs and so on.

Proposition 8. Let $G$ be a connected graph with $n$ vertices, $m$ edges and diameter $\operatorname{diam}(G)$. Then $4(n-1) m-M_{1}(G) \leq D^{\prime}(G) \leq-(\operatorname{diam}(G)-1) M_{1}(G)+2(n-$ 1) $m \operatorname{diam}(G)$ with either equality if and only if $\operatorname{diam}(G) \leq 2$.

Proof. Apply Lemma E(1) and Lemma E(2). 
Proposition 9. Let $G$ be a connected triangle- and quadrangle-free graph with $n \geq 3$ vertices, minimal degree $\delta$, maximal degree $\Delta, m$ edges and diameter $l$. Then

$$
\begin{aligned}
-(\Delta+2) M_{1}(G)+2 m[3(n-1)+\Delta] & \leq D^{\prime}(G) \\
& \leq-[l-1+\delta(l-2)] M_{1}(G)+2(l-2) m \delta \\
& +2(n-1) m l
\end{aligned}
$$

with right equality if and only if $G$ is a Moore graph of diameter 2, or a regular graph of diameter 3 (and girth 5, 6 or 7) and with left equality if and only if $G=S_{n}$, or $G$ is a Moore graph of diameter 2, or a regular graph of diameter 3 (and girth 5, 6 or 7).

Proof. Apply Lemma E(1) and Lemma E(3).

Proposition 10. Let $G$ be a connected graph with $n$ vertices, maximal degree $\Delta$ and $m$ edges. Then $-(\Delta+2) n(n-1)+2 m[3(n-1)+\Delta] \leq D^{\prime}(G)$ with equality if and only if $G$ is the star or a Moore graph of diameter 2.

Proof. Apply Lemma E(1) and Lemma E(4).

Proposition 11. Let $G$ be a connected graph with minimal degree $\delta$ and maximal degree $\Delta$. Then $2 \delta W(G) \leq D^{\prime}(G) \leq 2 \Delta W(G)$ with either equality if and only if $G$ is a regular graph.

Proof. Apply Lemma E(1) and Lemma E(5).

Proposition 12. Let $G$ be a tree with $n$ vertices and diameter $\operatorname{diam}(G)$. Then $D^{\prime}(G)=(n-1)[\operatorname{diam}(G)(2 m-2 n+2)-n]+4 W(G)$.

Proof. Apply Lemma E(1) and Lemma E(6).

\subsection{Reverse Degree Distance of Graphs}

The reverse degree distance of graphs is a new topological index proposed by Zhou and Trinajstić [21]. In the next two propositions, some extremal properties of this topological index are investigated.

Proposition 13. If $1 \leq n-1 \leq m$ and $G$ is a connected graph with $n$ vertices and $m$ edges, then ${ }^{r} D^{\prime}(G) \leq-4(n-1) m+\sum_{2}(n, m)+2(n-1) m \operatorname{diam}(G)$. Equality happens if and only if $G$ is a join of $K_{1}$ and a graph $G^{\prime}$ on $n-1$ vertices and $m-n+1$ edges with $\sum_{2}\left(G^{\prime}\right)=\sigma_{2}(n-1, m-n+1)$.

Proof. Apply Lemmas E(1) and F.

Proposition 14. Suppose $G$ is a connected graph with $n$ vertices and $m$ edges. Then for every $n \geq 3$ we have ${ }^{r} D^{\prime}(G) \leq-3 n^{2}+3 n+6+2 m(n-1) \operatorname{diam}(G)$ and the unique extremal graph is $K_{1, n-1}+e$.

Proof. Apply Lemmas E(1) and G(a). 
Proposition 15. Suppose $G$ is a connected graph with $n$ vertices and $m$ edges. Then for every $n \geq 4$ we have ${ }^{r} D^{\prime}(G) \leq-3 n^{2}-n+18+2 m(n-1) \operatorname{diam}(G)$ and the extremal graph is unique and may be obtained from $K_{1, n-1}$ by adding two edges having a common extremity.

Proof. Apply Lemmas E(1) and G(b).

\section{ACKNOWLEDGEMENT}

The authors are indebted to the referee for various valuable comments leading to improvements of the paper.

\section{REFERENCES}

[1] A. R. Ashrafi, T. Došlić, and A. Hamzeh, "The zagreb coindices of graph operations," Discrete Appl. Math., vol. 158, no. 15, pp. 1571-1578, 2010.

[2] A. R. Ashrafi, T. Došlić, and A. Hamzeh, "Extremal graphs with respect to the zagreb coindices," MATCH Commun. Math. Comput. Chem., vol. 65, no. 1, pp. 85-92, 2011.

[3] O. Bucicovschi and S. M. Cioabă, "The minimum degree distance of graphs of given order and size," Discrete Appl. Math., vol. 156, no. 18, pp. 3518-3521, 2008.

[4] K. C. Das and I. Gutman, "Some properties of the second zagreb index," MATCH Commun. Math. Comput. Chem., vol. 52, pp. 103-112, 2004.

[5] M. V. Diudea, I. Gutman, and L. Jäntschi, Molecular Topology, ser. Huntington. New York: Nova Science, 2001.

[6] A. A. Dobrynin and A. A. Kochetova, "Degree distance of agraph: A degree analogue of the wiener index," J. Chem. Inf. Comput. Sci., vol. 34, no. 5, pp. 1082-1086, 1994.

[7] T. Došlić, "Vertex-weighted wiener polynomials for composite graphs," Ars Math. Contemp., vol. 1, no. 1, pp. 66-80, 2008.

[8] I. Gutman, "Selected properties of the schultz molecular topological index," J. Chem. Inf. Comput. Sci., vol. 34, no. 5, pp. 1087-1089, 1994.

[9] I. Gutman and K. C. Das, "The first zagreb index 30 years after," MATCH Commun. Math. Comput. Chem., vol. 50, pp. 83-92, 2004.

[10] A. Ilić and D. Stevanović, "On comparing zagreb indices," MATCH Commun. Math. Comput. Chem., vol. 62, no. 3, pp. 681-687, 2009.

[11] A. Ioan Tomescu, "Unicyclic and bicyclic graphs having minimum degree distance," Discrete Appl. Math., vol. 156, no. 1, pp. 125-130, 2008.

[12] M. H. Khalifeh, H. Yousefi-Azari, and A. R. Ashrafi, "The first and second zagreb indices of some graph operations," Discrete Appl. Math., vol. 157, no. 4, pp. 804-811, 2009.

[13] M. H. Khalifeh, H. Yousefi-Azari, A. R. Ashrafi, and S. G. Wagner, "Some new results on distancebased graph invariants," European J. Combin., vol. 30, no. 5, pp. 1149-1163, 2009.

[14] M. Liu and B. Liu, "New sharp upper bounds for the first zagreb index," MATCH Commun. Math. Comput. Chem., vol. 62, no. 3, pp. 689-698, 2009.

[15] M. J. Nadjafi-Arani, G. H. Fath-Tabar, and A. R. Ashrafi, "Extremal graphs with respect to the vertex pi index," Appl. Math. Lett., vol. 22, no. 12, pp. 1838-1840, 2009.

[16] S. Nikolić, G. Kovačević, A. Miličević, and N. Trinajstić, "The zagreb indices 30 years after," Croat. Chem. Acta, vol. 76, no. 2, pp. 113-124, 2003.

[17] L. Sun and S. Wei, "Comparing the zagreb indices for connected bicyclic graphs," MATCH Commun. Math. Comput. Chem., vol. 62, no. 3, pp. 699-714, 2009.

[18] D. B. West, Introduction to graph theory. Upper Saddle River: Prentice Hall, 1996. 
[19] L. Zhang and B. Wu, "The nordhaus-goddum-type inequalities for some chemical indices," MATCH Commun. Math. Comput. Chem., vol. 54, no. 1, pp. 189-194, 2005.

[20] B. Zhou and I. Gutman, "Relations between wiener, hyper-wiener and zagreb indices," Chem. Phys. Lett., vol. 394, no. 1-3, pp. 93-95, 2004.

[21] B. Zhou and N. Trinajstić, "On reverse degree distance," J. Math. Chem., vol. 47, no. 1, pp. 268275, 2010.

Author's address

S. Hossein-Zadeh, A. Hamzeh and A. R. Ashrafi

Department of Mathematics, Faculty of Science, University of Kashan, Kashan 87317-51167, Iran

School of Mathematics, Institute for Research in Fundamental Sciences (IPM), P. O. Box: 193955746, Tehran, Iran

E-mail address: ashrafi@kashanu.ac.ir 